Check for updates

Cite this: RSC Adv., 2020, 10, 44834

DOI: 10.1039/d0ra90132f

rsc.li/rsc-advances

\section{Correction: Direct electrodeposition of cationic pillar[6]arene-modified graphene oxide composite films and their host-guest inclusions for enhanced electrochemical performance}

\author{
Qunpeng Duan, ${ }^{\text {*a }}$ Lijie Wang, ${ }^{a}$ Fei Wang, ${ }^{a}$ Hongsong Zhang ${ }^{a}$ and Kui Lu*ab \\ Correction for 'Direct electrodeposition of cationic pillar[6]arene-modified graphene oxide composite films \\ and their host-guest inclusions for enhanced electrochemical performance' by Qunpeng Duan et al., RSC \\ Adv., 2020, 10, 21954-21962, DOI: 10.1039/DORA03138K.
}

The authors regret omitting a citation of their related paper published in Frontiers in Chemistry: 'Facile one-step electrodeposition preparation of cationic pillar[6]arene-modified graphene films on glassy carbon electrodes for enhanced electrochemical performance' (DOI: 10.3389/fchem.2020.00430) shown as ref. 1 here. The citation should appear as ref. 58 in the original article. ${ }^{1}$

The authors regret that it was not clear in the original article that the ErGO-CP6/GCE film had been previously reported by them in their related Frontiers in Chemistry paper ${ }^{1}$ and therefore the sentence at the start of paragraph 3 on page 2 'In this work, we report for the first time preparation of CP6 functionalized graphene films on glassy carbon electrode (GCE) directly from GO-CP6 dispersions by facile one-step pulsed electrodeposition technique (Scheme 1).' should be 'In this work, we report the preparation of CP6 functionalized graphene films on glassy carbon electrode (GCE) directly from GO-CP6 dispersions by facile one-step pulsed electrodeposition technique (Scheme 1), which was previously reported by us. ${ }^{58}$.

The authors also wish to clarify the differences between this RSC Advances paper and the Frontiers in Chemistry paper. ${ }^{1}$ The papers use different guests molecules and different optimum pulse electrodeposition parameters and the RSC Advances paper reports an improvement in electrochemical performance with additional characterisation, stability studies and the analysis of real samples which are not reported in the Frontiers in Chemistry paper. ${ }^{1}$

The appropriate figure captions have been updated to reflect the data reproduced from the Frontiers in Chemistry paper. ${ }^{1}$

Scheme 1 Schematic illustration for the pulsed electrodeposition preparation of ErGO and ErGO-CP6 films on the surface of GCE and sensing the guest molecules by an electrochemical strategy. Reproduced with permission from ref. 1. Copyright 2020 Frontiers.

Fig. 1 Characterization of materials. FTIR spectra (A), UV-vis absorption spectra (B), TGA curves of CP6, GO-CP6, and GO (C), and XPS survey spectra of GO and GO-CP6 (D). The data in (a, $c$ and d) have been reproduced with permission from ref. 1. Copyright 2020 Frontiers.

Fig. 4 (A) Raman spectra of GO and ErGO. (B) Raman spectra of GOCP6 and ErGO-CP6. Reproduced with permission from ref. 1. Copyright 2020 Frontiers.

\title{
References
}

1 Q. Duan, L. Wang, F. Wang, H. Zhang and K. Lu, Front. Chem., 2020, 8, 430.

${ }^{a}$ School of Materials and Chemical Engineering, Henan University of Engineering, Zhengzhou, 450006, China. E-mail: qpduan@haue.edu.cn ${ }^{b}$ School of Chemical Engineering and Food Science, Zhengzhou Institute of Technology, Zhengzhou, 450044, China. E-mail: luckyluke@haue.edu.cn 